\title{
Popsicle-Stick Cobra Wave
}

\author{
Jean-Philippe Boucher, ${ }^{1}$ Christophe Clanet, ${ }^{1}$ David Quéré, ${ }^{2}$ and Frédéric Chevy ${ }^{3}$ \\ ${ }^{1}$ LadHyX, UMR 7646 du CNRS, École Polytechnique, 91128 Palaiseau Cedex, France \\ ${ }^{2}$ PMMH, UMR 7636 du CNRS, ESPCI, 75005 Paris, France \\ ${ }^{3}$ Laboratoire Kastler Brossel, ENS-PSL Research University, CNRS, \\ UPMC, Collège de France, 24, rue Lhomond, 75005 Paris, France
}

(Received 23 February 2017; published 25 August 2017)

\begin{abstract}
The cobra wave is a popular physical phenomenon arising from the explosion of a metastable grillage made of popsicle sticks. The sticks are expelled from the mesh by releasing the elastic energy stored during the weaving of the structure. Here we analyze both experimentally and theoretically the propagation of the wave front depending on the properties of the sticks and the pattern of the mesh. We show that its velocity and its shape are directly related to the recoil imparted to the structure by the expelled sticks. Finally, we show that the cobra wave can only exist for a narrow range of parameters constrained by gravity and rupture of the sticks.
\end{abstract}

DOI: 10.1103/PhysRevLett.119.084301

The physics of metastable states is a classical topic of statistical physics [1,2]. A well-known route to relax towards equilibrium is via a nonlinear front that propagates with a constant speed, such as in viral spread [3,4], biochemical reactions [5], or combustion [6,7]. In mechanics, the domino race provides an example of such a process for a nonconnected network [8,9]. For entangled structures, the question of the optimization of the strength of grillages has been addressed $[10,11]$ especially because of its role in construction [12], but their stability remains an open question. The same type of question also arises in biological systems, such as in the microtubule catastrophe $[13,14]$. Microtubules are assemblies of GDP tubulin arranged in a tubular shape ending with a cap of GTP tubulin. The loss of this cap triggers a rapid depolymerization driven by the release of the stored mechanical strain [15-17]. Here we study a macroscopic version of such a system, namely, the so-called "popsicle-stick cobra wave" [18], obtained by releasing a mesh of sticks woven according to Fig. 1(b).

To generate a cobra wave, the whole structure is loaded by the geometrically constrained bending of the individual sticks and is held together by the red and blue sticks at the end of the mesh [see Fig. 1(b)]. When one of them is removed, the structure unravels by expelling one by one the freed sticks. Because of the asymmetry of the weaving and the presence of the ground, two very different dynamics occur depending on which stick was initially removed. When the red stick is taken away first, the sticks are expelled upwards and by reaction they pin down the rest of the mesh to the ground (see movie Inverse-Cobra-Wave in the Supplemental Material [19]). The outcome is dramatically different when the blue stick is removed. In this case, the sticks are expelled downwards and they raise the whole structure as presented in Fig. 2(a) (see also the movie Cobra-Wave provided in Ref. [19]). After a few hundreds of $\mathrm{ms}$, the shape of the wave reaches a steady state

[Fig. 2(b)], and propagates at a few meters per second. Both the shape and the velocity remain the same until the wave front reaches the end of the grillage [Fig. 2(c)]. In this Letter, we combine experimental and theoretical approaches to characterize the velocity and the shape of the cobra wave in the steady state.

Since the lifting force raising the lattice originates from the recoil imparted by the expelled sticks, the global dynamics of the wave is set by the ejection rate $\gamma$ and the momentum transferred during the expulsion $M v$, where $M$ is the mass of a stick and $v$ the velocity of a stick right after expulsion. The time $\gamma^{-1}$ taken by a stick to exit the mesh is given by $L / v$, where $L$ is the length of the stick [Fig. 1(a)]. Taking $E$ the Young's modulus, $w$ the width, and $e$ the thickness of individual sticks [Fig. 1(a)], $v$ can be estimated from the balance between the kinetic energy $M v^{2}$

(a)

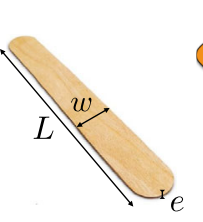

(c)
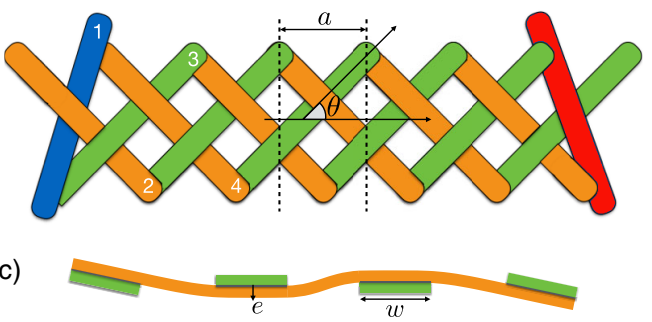

FIG. 1. (a) Picture of a wooden stick with its characteristic parameters: length $L$, width $w$, thickness $e$, mass $M$, density $\rho$ and Young's modulus $E$. (b) Schematics of the lattice with definition of the angle $\theta$ of the lattice and the spatial period $a$ of the pattern. The blue and red sticks are the sticks that end the lattice. The construction of the lattice starts with the blue stick, then the sticks are added one after the other according to the numbering for the first four sticks. (c) Schematic side view of a stick deformed in the mesh. 

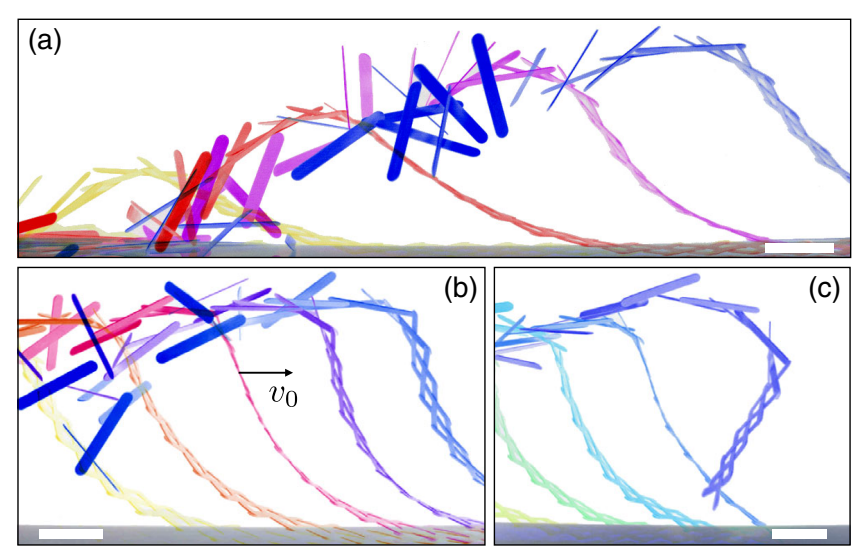

FIG. 2. Time-lapse photographs of the cobra wave obtained with sticks of type 1 (see the Supplemental Material [19]) for $\theta=45^{\circ}$, from videos taken at $1000 \mathrm{fps}$ with a Photron-Fastcam high-speed camera. The different colors represent the wave at different instants. (a) At the beginning ( $\Delta t=100 \mathrm{~ms}$ between two consecutive images); (b) during the stationary phase ( $\Delta t=70 \mathrm{~ms}$ ), with $v_{0} \simeq 2.2 \mathrm{~m} / \mathrm{s}$ the velocity of the wave front; (c) at the end $(\Delta t=70 \mathrm{~ms})$. Scale bars are $10 \mathrm{~cm}$ long.

of a stick after ejection and the bending energy $E_{\mathrm{el}} \sim$ $E w e^{5} / L^{3}$ stored in each stick blocked by the lattice [this expression is obtained by noting that the curvature of a stick imposed by its neighbors is $\Gamma \sim e / L^{2}$, see Fig. 1(c)].

From the previous scaling analysis, we readily deduce the velocity $v_{0}$ of the wave front. Indeed, since the sticks are expelled one by one, we have $v_{0}=a \gamma / 2$, where $a$ is the spatial period of the pattern [Fig. 1(b)]. Noting that, up to a geometric factor depending on the angle $\theta$, we have $a \propto L$, both $v$ and $v_{0}$ scale as

$$
v \sim v_{0}=b(\theta) \sqrt{\frac{E}{\rho}}\left(\frac{e}{L}\right)^{2},
$$

where $\rho$ is the mass density of a stick and $b(\theta)$ is a scaling factor that depends on the geometry of the mesh. With $c=$ $\sqrt{E / \rho}$ the speed of sound in the material, we find that $v_{0} \propto c(e / L)^{2}$. In Fig. 3, we confirm experimentally this scaling for six kinds of wooden sticks (the values of the mechanical and geometric parameters of the different stick models are given in Ref. [19]) and we observe that indeed $v \propto v_{0}$. As expected, the speed does not depend on the width of the sticks and increases quadratically with the ratio $e / L$. In Fig. 3(i), one can see that the speed of the cobra decreases with the angle of the lattice $\theta$. This trend can be easily understood qualitatively by noting that the velocity of the wave is proportional to the spatial periodicity $a=L \cos \theta / 3$.

We now focus on the shape of the wave. The height of the cobra can be understood quantitatively within a generalized version of Euler's elastica theory, where the mesh profile results from a competition between elasticity,

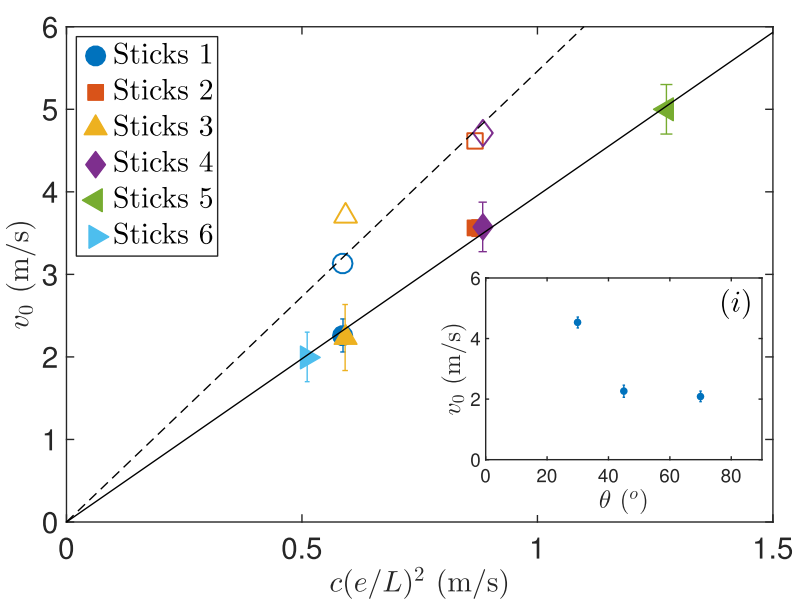

FIG. 3. Speed of the wave front $v_{0}$ (solid dots) and speed of the expelled sticks $v$ (open dots) as a function of a characteristic speed $c(e / L)^{2}$ for $\theta=45^{\circ}$ and six different kinds of sticks (see the Supplemental Material [19]). The black line corresponds to the fit $v_{0}=3.95 c(e / L)^{2}$ and the dashed line to the fit $v=5.46 c(e / L)^{2}$. (i) Speed of the cobra wave $v_{0}$ as a function of the angle of the lattice $\theta$ for sticks of type 1 .

gravity, and recoil imparted by the expelled sticks [20]. We treat the mesh as a linear continuous medium characterized by a flexion modulus $\bar{K}[19]$ and we describe the expulsion of the sticks by a force $\boldsymbol{F}_{0}$ and a torque $\boldsymbol{C}_{0}$ exerted at the free end of the grillage. We further assume that the friction between sticks prevents the deformation of the lattice, allowing us to describe the mesh as an inextensible linear medium described by a profile $\boldsymbol{r}(s, t)$, where $s$ is the curvilinear abscissa (Fig. 4), the local force $\boldsymbol{F}(s, t)$ and torque $\boldsymbol{C}(s, t)$ are given by

$$
\boldsymbol{F}=-\bar{K} \partial_{s}^{3} \boldsymbol{r}, \quad \boldsymbol{C}=\bar{K} \partial_{s} \boldsymbol{r} \times \partial_{s}^{2} \boldsymbol{r} .
$$

In the steady state, the shape of the cobra is constant and moves at the velocity $v_{0}$. We therefore have $\boldsymbol{r}(s, t)=$ $\boldsymbol{r}\left(s^{\prime}=s-v_{0} t\right)$ and writing Newton's law for an infinitesimally small element of the mesh leads to the following dynamical equation,

$$
\mu v_{0}^{2} \partial_{s^{\prime}}^{2} \boldsymbol{r}=\mu \boldsymbol{g}-\bar{K} \partial_{s^{\prime}}^{4} \boldsymbol{r}+\partial_{s^{\prime}}(T \boldsymbol{\tau})+\boldsymbol{R},
$$

where $\mu=2 M / a$ is the linear mass density of the cobra, $T$ the longitudinal tension, $\boldsymbol{\tau}$ the tangent unit vector, and $\boldsymbol{R}$ the ground reaction. We assume that the contact with the ground occurs for $s^{\prime} \leq 0$, so that $z\left(s^{\prime} \leq 0\right)=0$ and $\boldsymbol{R}\left(s^{\prime} \geq 0\right)=0$, and $s_{\max }$ is the total mesh length rising above the ground. Projecting Eq. (3) on the tangent and normal directions, these equations can be recast into a closed equation for the curvature $\Gamma=\left|\partial_{s}^{2} \boldsymbol{r}\right|$,

$$
\frac{1}{2}\left(\frac{d^{3} \Gamma^{2}}{d \alpha^{3}}+\frac{d \Gamma^{2}}{d \alpha}\right)=\frac{\mu g}{\bar{K}}\left(\frac{2 \sin \alpha}{\Gamma}+\frac{\cos \alpha}{\Gamma^{2}} \frac{d \Gamma}{d \alpha}\right),
$$




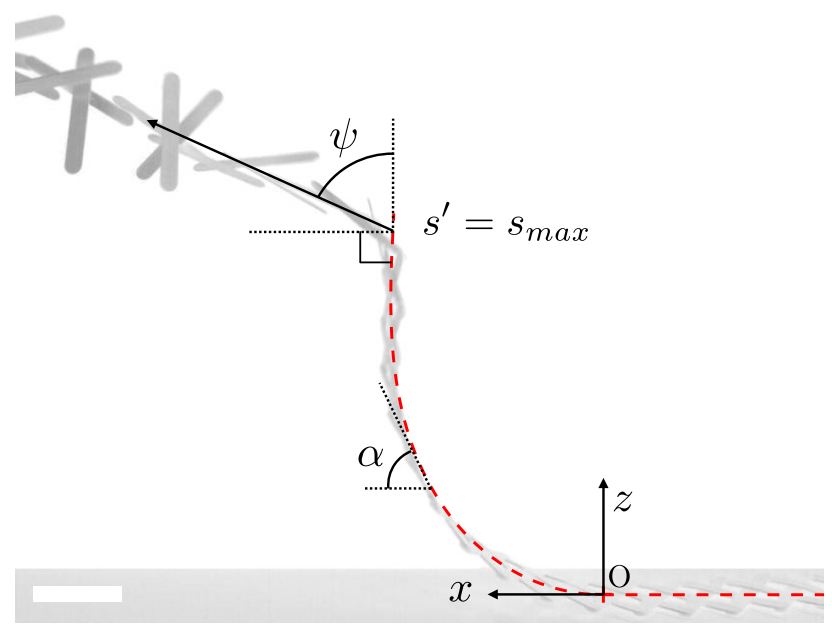

FIG. 4. Experimental cobra profile for sticks of type 2. The steady profile (red dashed line) is described theoretically by a parametric curve $\boldsymbol{r}\left(s^{\prime}\right)$, where $s^{\prime}$ is the curvilinear abscissa. $\alpha$ is the angle between the mesh and the horizontal axis, and $\psi$ the angle between the velocity of the expelled sticks and the tangent vector in $s^{\prime}=s_{\max }$. The scale bar is $10 \mathrm{~cm}$ long.

where $\alpha$ is the local angle between the mesh and the horizontal axis.

This equation is of third order in $\alpha$ and thus requires three boundary conditions to be solved. We obtain these conditions by writing the stress at the free end $\alpha_{\max }=\alpha\left(s_{\max }\right)$ of the mesh, namely,

$$
\begin{aligned}
& C_{0}=\bar{K} \Gamma, \\
& F_{\|}=T+\bar{K} \Gamma^{2}, \\
& F_{\perp}=-\frac{K}{2} \frac{d \Gamma^{2}}{d \alpha},
\end{aligned}
$$

where $F_{\|}=\boldsymbol{F}_{0} \cdot \boldsymbol{\tau}$ and $F_{\perp}=\boldsymbol{F}_{0} \cdot \boldsymbol{n}$ and the right-hand side terms are taken at $\alpha=\alpha_{\max }$. A fourth condition is required by the fact that, contrary to the elastica problem where the length of the beam is fixed, we must here determine self-consistently the mesh length rising above the ground. To close the system, we, therefore, impose the usual mobile contact-point condition $\Gamma(\alpha=0)=0$ that assumes that there is no adhesion energy between the mesh and the ground [21].

On the one hand, the forces can be calculated from the momentum transfer between the lattice and the expelled sticks and we have $F_{\perp}=\mu v_{0} v \sin \psi$ and $F_{\|}=$ $\mu v_{0}\left(v_{0}-v \cos \psi\right)$.

On the other hand, the torque exerted at the free end of the cobra can be neglected. Indeed, assuming that all the elastic energy $E_{\text {el }}$ is converted into rotational energy of the sticks $E_{\mathrm{rot}}=\frac{1}{2} I \omega^{2}$ with $I$ the moment of inertia of a stick and $\omega$ its angular velocity, the angular momentum of an expelled stick is $\mathcal{L}=I \omega=\sqrt{2 I E_{\mathrm{el}}}$. Since the torque is
$C=(d \mathcal{L} / d t) \sim \gamma \mathcal{L}$, we get the upper bound $C_{\max } \sim \gamma \sqrt{2 I E_{\mathrm{el}}}$. In Eq. (6), the torque is compared to $\bar{K} \Gamma$. Using Eq. (5), we can eliminate $\Gamma$ and we see that the relative importance of the torque and the force is driven by the dimensionless number

$$
\frac{C^{2}}{\bar{K} F} \simeq \frac{\gamma^{2} I\left(K e^{2} / L^{3}\right)}{K \gamma M v_{0}} \simeq\left(\frac{e}{L}\right)^{2} \ll 1
$$

where $K \sim \bar{K}$ is the flexion modulus of a single stick [19] and we have used the fact that $v \sim v_{0} \sim \gamma L$ and $I \sim M L^{2}$. We thus see that for thin sticks, the torque does not affect much of the shape of the cobra.

Equation (4) can be solved numerically in the general case using the shooting method and the height of the cobra can be obtained from

$$
H=\int_{0}^{\alpha_{\max }} \frac{\sin \alpha}{\Gamma(\alpha)} d \alpha
$$

The analysis of Eqs. (4)-(9) shows that $H$ follows the general scaling

$$
H=\sqrt{\frac{\bar{K}}{\mu v_{0} v}} h_{\psi}(\Lambda), \quad \text { with } \quad \Lambda=g \sqrt{\frac{\bar{K}}{\mu v_{0}^{3} v^{3}}} .
$$

When gravity can be neglected, Eq. (4) can be solved analytically and yields $\Gamma=\Gamma_{0} \sqrt{\cos (\psi-\alpha)-\cos (\psi)}$ with $\Gamma_{0}=\sqrt{2 \mu v v_{0} / \bar{K}}$. We then obtain $\alpha_{\max }=2 \psi$ and the dimensionless height can be expressed in terms of the elliptic integrals $\mathcal{E}$ and $\mathcal{K}$ [22] with

$$
h_{\psi}(0)=2 \sin (\psi)\{2 \mathcal{E}[\sin (\psi / 2)]-\mathcal{K}[\sin (\psi / 2)]\} .
$$

For large values of $\Lambda$, gravity becomes dominant and the cobra does not rise as high. In this regime, $\alpha_{\max } \rightarrow 0$ and we can therefore neglect the lower order derivatives in each sides of Eq. (4) leading to the simplified expression

$$
\frac{d^{3} \Gamma^{2}}{d \alpha^{3}}=\frac{2 \mu g}{\bar{K} \Gamma^{2}} \frac{d \Gamma}{d \alpha} .
$$

This equation can be solved analytically leading to an asymptotic behaviour $h_{\psi} \simeq 2 \sin ^{4} \psi / 3 \Lambda^{3}$.

The asymptotic behaviors obtained in both the weak and strong gravity regimes can be understood by a straightforward argument. We note first that Eqs. (7) and (8) lead to the following scalings:

$$
F_{\perp} \simeq \bar{K} \frac{\Gamma_{0}^{2}}{\alpha_{\max }}, \quad H \simeq \frac{\alpha_{\max }^{2}}{\Gamma_{0}} .
$$

We can then distinguish two regimes. For small $g$, the height is saturated and $\alpha_{\max } \simeq 1$, hence, $\Gamma_{0} \simeq \sqrt{F_{\perp} / \bar{K}}$ and 


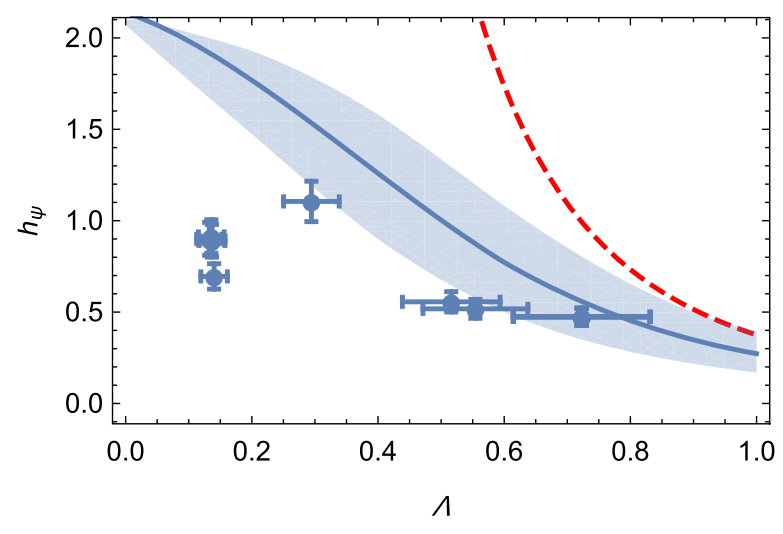

FIG. 5. Dimensionless height $h_{\psi}$ as a function of the dimensionless number $\Lambda=g \sqrt{\bar{K} /\left(\mu v_{0}^{3} v^{3}\right)}$ which compares gravity and elasticity, for the six different kinds of sticks (see the Supplemental Material [19]). The solid line corresponds to the prediction of Eqs. (4)-(9) for $\psi=60^{\circ}$. The shaded band corresponds to the observed $10^{\circ}$ variations of the ejection angle. The red dashed line represents the large $\Lambda$ expansion $h_{\psi} \simeq 2 \sin ^{4} \psi / 3 \Lambda^{3}$ for $\psi=60^{\circ}$.

$H \simeq \Gamma_{0}^{-1} \simeq \sqrt{\bar{K} / F_{\perp}}$. The scaling for $H$ yields the condition $h_{\psi} \simeq 1$ for weak gravity. Using Eq. (1), we can express $v_{0}$ and $F_{\perp}$ with $K, e$, and $L$. We then obtain a simple scaling for $H \simeq L^{2} / e$, which does not depend any more on the elasticity of the mesh. This purely geometric scaling stems from the fact that, when gravity is negligible, stick elasticity provides both the thrusting and restoring forces responsible for the shape of the mesh.

For heavy sticks, the lattice is almost horizontal and the value of $\alpha_{\max }$ is set by the balance between $F_{\perp}$ and the weight. The length of the cobra being $s_{\max } \simeq \alpha_{\max } / \Gamma_{0}$, we have thus the additional condition

$$
F_{\perp} \simeq \frac{\mu g \alpha_{\max }}{\Gamma_{0}} .
$$

Combining Eqs. (11) and (12) yields the condition $\alpha_{\max } \simeq$ $\Lambda^{-2}$ and $h_{\psi} \simeq 1 / \Lambda^{3}$. The transition between the two regimes occurs for $\Lambda \simeq 1$.

We now compare the previous model to our measurements. We measured the velocity $v$ and the angle $\psi$ at which the sticks are expelled. We observe that for almost all stick models, $\psi$ varies between $50^{\circ}$ and $70^{\circ}$. In Fig. 5, we compare our measurements to the predicted value $h_{\psi=60^{\circ}}$ without any adjustable parameter (note in particular that the effective flexion modulus of the mesh is measured independently as described in Ref. [19]). Except in the weakgravity regime, we observe a relatively good agreement between experiment and theory. We attribute the saturation of the height of the cobra wave for small $\Lambda$ to the strong curvature of the mesh (in this regime the radius of curvature is only a few times larger than stick length), leading to a breakdown of the underlying assumptions of the theoretical

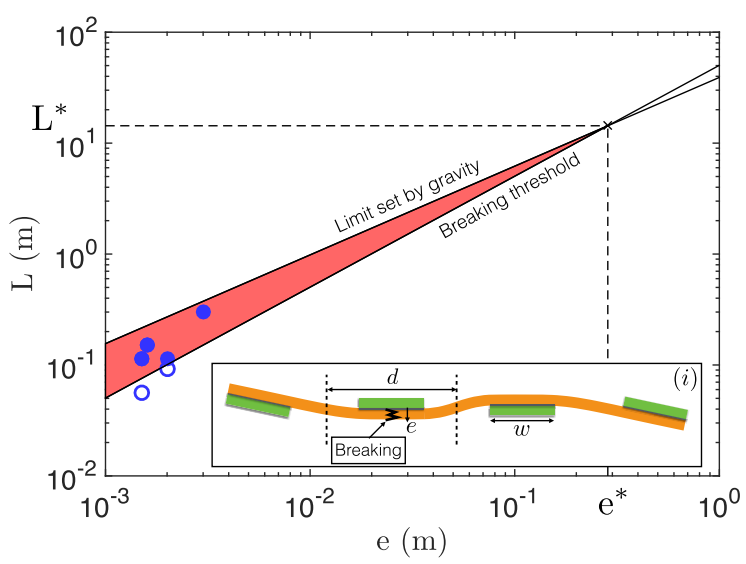

FIG. 6. Set of parameters $(e, L)$ for which a cobra stick wave can be observed (red region). This region is limited by two conditions given in Eqs. (13) and (15): the limit set by gravity $L_{\max }=$ $\left(36 E e^{4} / \rho g\right)^{1 / 5}$ and the breaking limit $L_{\min }=1.5 \times 3 \sqrt{E / \sigma^{*}} e$. Solid blue dots: sets of parameters for which the cobra-stick wave is observed. Open blue dots: sets of parameters for which the cobra-stick wave could not be observed (the sticks are too small and therefore break). (i) Schematics of the shape of a stick in the lattice with the most probable breaking region.

model. For instance, the validity of the continuum approximation for the description of the mesh, or the linear approximation for the bending energy. Friction can also play a larger role, and the strong deformation can weaken the structure, preventing it from reaching its predicted height.

Finally, we discuss the condition of existence of the cobra wave. The first requirement is that the curvature energy stored in a single stick $\left(E_{\mathrm{el}}=18 E w e^{5} / L^{3}\right)$ should overcome the gravitational energy $\left(E_{g}=\rho g w e L^{2} / 2\right)$. This leads to an upper bound for the length $L$ of the sticks:

$$
L<L_{\max }=\left(\frac{36 E e^{4}}{\rho g}\right)^{1 / 5} .
$$

However, the length $L$ of the sticks cannot be too small because if so it becomes impossible to build the lattice: the sticks either break or slide over each other destroying the lattice. The breaking condition is derived from a simple scaling law for the bending stress in a beam that sets an upper limit for the curvature of a stick in the lattice [Fig. 6(i)],

$$
\mathcal{C} \sim \frac{e}{d^{2}}<\frac{\sigma^{*}}{E e},
$$

where the length $d=(L-w) / 3$ is defined in Fig. 6(i) and $\sigma^{*}$ is the bending stress at rupture of the material. We then get a lower bound for the length $L$ of the sticks

$$
L>L_{\min } \sim 3 \sqrt{\frac{E}{\sigma^{*}}} e+w .
$$


For wooden sticks, these two conditions set the boundaries of the cobra-wave region of existence. The phase diagram $(e, L)$ is plotted in Fig. 6 with the region of existence of the cobra stick wave in red, assuming the width $w$ to be negligible compared to the length $L$ of the sticks.

In conclusion, we have shown that the shape of the popsicle-stick cobra wave was the result of a competition between the thrust provided by the expulsion of the sticks and the elastic and gravitational restoring forces. Depending on the relative importance of gravity, we identified two asymptotic regimes. In particular, for negligible gravity, the cobra rises at a height which is solely set by the weaving pattern and the dimensions of single sticks. Finally, we showed that the Cobra wave can only exist in a narrow region of the parameter space bounded by gravity and rupture of the sticks.

This work was inspired by one of the problems of the International Physicists' Tournament (IPT). The authors acknowledge fruitful discussions with Basile Audoly, Daniel Suchet, and the Ecole normale supérieure and École Polytechnique IPT teams.

[1] L. D. Landau and E. M. Lifshitz, Statistical Physics, Vol. 5 (Pergamon Press, New York, 1980).

[2] J. S. Langer, Statistical theory of decay of metastable states, Ann. Phys. (N.Y.) 54, 258 (1969).

[3] R. A. Fisher, The wave of advance of advantageous genes, Ann. Hum. Genet. 7, 355 (1937).

[4] A. Kolmogoroff, I. Petrovsky, and N. Piscounoff, Study of the diffusion equation with growth of the quantity of matter and its application to a biological problem, Moscow Univ. Math. Bull. 1, 1 (1937).

[5] J. D. Murray, Mathematical Biology (Springer, New York, 2000).

[6] Ya. B. Zeldovich, G. I. Barenblatt, V. B. Librovich, and G. M. Makhviladze, The Mathematical Theory of Combustion and Explosions (Consultants Bureau, New York, 1985).

[7] P. Clavin and G. Searby, Combustion Waves and Fronts in Flows (Cambridge University Press, Cambridge, England, 2016).
[8] W. J. Stronge, The domino effect: A wave of destabilizing collisions in a periodic array, Proc. R. Soc. A 409, 199 (1987)

[9] W. J. Stronge and D. Shu, The domino effect: Successive destabilization by cooperative neighbours, Proc. R. Soc. A 418, 155 (1988).

[10] G. I. N. Rozvany, Grillages of maximum strength and maximum stiffness, Int. J. Mech. Sci. 14, 651 (1972).

[11] T. Tarnai, Duality between plane trusses and grillages, Int. J. Solids Struct. 25, 1395 (1989).

[12] P. M. Stylianidis, D. A. Nethercot, B. A. Izzuddin, and A. Y. Elghazouli, Robustness assessment of frame structures using simplified beam and grillage models, Engineering structures 115, 78 (2016).

[13] I. M. Cheeseman and A. Desai, Molecular architecture of the kinetochore-microtubule interface, Nat. Rev. Mol. Cell Biol. 9, 33 (2008).

[14] A. Desai and T. Mitchison, Microtubule polymerization dynamics, Annu. Rev. Cell Dev. Biol. 13, 83 (1997).

[15] T. Mitchison and M. Kirschner, Dynamic instability of microtubule growth, Nature (London) 312, 237 (1984).

[16] T. Mitchison and M. Kirschner, Microtubule assembly nucleated by isolated centrosomes, Nature (London) 312, 232 (1984).

[17] H. Bowne-Anderson, A. Hibbel, and J. Howard, Regulation of microtubule, growth and catastrophe: Unifying theory and experiment, Trends Cell Biol. 25, 769 (2015).

[18] https://en.wikipedia.org/wiki/Stick_bomb.

[19] See Supplemental Material at http://link.aps.org/ supplemental/10.1103/PhysRevLett.119.084301 containing two movies describing the dynamics of the mesh, as well as the physical parameters of the sticks used in our study and the experimental characterization of the effective modulus of the mesh.

[20] B. Audoly and Y. Pomeau, Elasticity and Geometry: From Hair Curls to the Non-Linear Response of Shells (Oxford University Press, New York, 2010).

[21] R. Burridge and J. B. Keller, Peeling, slipping and cracking - some one-dimensional free-boundary problems in mechanics, SIAM Rev. 20, 31 (1978).

[22] M. Abramowitz and I. A. Stegun, Handbook of Mathematical Functions: With Formulas, Graphs, and Mathematical Tables (Courier Corporation, 1964). 